BI-TP 2010/41

\title{
Effective Polyakov-loop theory for pure Yang-Mills from strong coupling expansion
}

\author{
Jens Langelage* \\ Fakultät für Physik, Universität Bielefeld, \\ 33501 Bielefeld, Germany \\ E-mail: jlangephysik.uni-bielefeld.de

\section{Stefano Lottini*, Owe Philipsen} \\ Institut für Theoretische Physik - Johann Wolfgang Goethe-Universität \\ Max-von-Laue-Str. 1, 60438 Frankfurt am Main, Germany \\ E-mail: lottini, philipsen ath.physik.uni-frankfurt.de
}

\begin{abstract}
Lattice Yang-Mills theories at finite temperature can be mapped onto effective $3 \mathrm{~d}$ spin systems, thus facilitating their numerical investigation. Using strong-coupling expansions we derive effective actions for Polyakov loops in the $S U(2)$ and $S U(3)$ cases and investigate the effect of higher order corrections. Once a formulation is obtained which allows for Monte Carlo analysis, the nature of the phase transition in both classes of models is investigated numerically, and the results are then used to predict - with an accuracy within a few percent - the deconfinement point in the original $4 \mathrm{~d}$ Yang-Mills pure gauge theories, for a series of values of $N_{\tau}$ at once.
\end{abstract}

The XXVIII International Symposium on Lattice Field Theory

June 14-19,2010

Villasimius, Sardinia Italy

\footnotetext{
${ }^{*}$ Speaker.
} 


\section{Introduction}

In the framework of non-abelian gauge theories at finite temperature, several effective descriptions have been pursued in order to overcome the infrared problems [1] connected with perturbative approaches to the fundamental theory. A rather successful technique is dimensional reduction [2, 3]. Thanks to the presence of different energy scales, induced by the finite temperature dynamics of the original $(3+1)$-dimensional theory, an integration over the hard modes leads to a $3 \mathrm{~d}$ effective description which can then be solved in a non-perturbative way (e. g. by Monte Carlo integration).

In the case of QCD, this technique loses its validity in the confined phase; however, one would want to devise effective methods to study the vicinity of the deconfinement transition: this is not a completely trivial task since the standard perturbative dimensional reduction does not retain the $Z(N)$ symmetry of the original Yang-Mills theory [ [ $₫$ ]. One can then follow a different strategy, namely writing down a general theory respecting the desired symmetry and then fixing the (many) couplings by matching with particular observables [5], 6]. While for $S U(2)$ the phase transition is captured correctly by such approaches [7, 8], for the physically relevant $S U(3)$ gauge theory a satisfactory fixing of all couplings is still an open issue.

A different way to pin down a $3 \mathrm{~d}$ effective theory is to employ lattice strong coupling expansions. This idea, first considered in [9], has been pursued by various authors [10, 11, 12, 13, 14, 15] and leads to theories with Polyakov loops as fundamental degrees of freedom. The contribution from spatial plaquettes was often neglected, a simplification which preserves the universal behaviour of the theory; in [16], instead, they were explicitly taken into account. Recent developments including staggered fermions can be found in [17.

The models proposed here systematically extend this approach by providing series for the effective couplings up to a certain order and are thus valid beyond the spatial strong coupling limit. As is to be expected from strong-coupling expansions, our results will have a finite radius of convergence, which is supposed to coincide with the deconfinement transition: in this sense, our effective formulation is complementary to weak coupling approaches. The effective actions we propose are subsequently studied by means of Monte Carlo integration, and the results are shown to lead to the correct order of the transition as well as to good estimates of the deconfinement point.

\section{Derivation of the effective theory}

\subsection{General strategy and $S U(2)$}

Consider the partition function of a $(3+1)$-dimensional lattice gauge field theory at finite temperature $\left(T=\frac{1}{a N_{\tau}}\right)$ with gauge group $S U(N)$ and Wilson's gauge action

$$
Z=\int\left[d U_{0}\right]\left[d U_{i}\right] \exp \left[\frac{\beta}{2 N} \sum_{p}\left(\operatorname{tr} U_{p}+\operatorname{tr} U_{p}^{\dagger}\right)\right], \quad \beta=\frac{2 N}{g^{2}} .
$$

Finite temperature and the bosonic nature of the degrees of freedom imply the use of periodic boundary conditions in the time direction. 
In order to arrive at an effective three-dimensional theory, we integrate out the spatial degrees of freedom and get schematically [13]

$$
\begin{aligned}
Z & =\int\left[d U_{0}\right] \exp \left[-S_{\text {eff }}\right] \\
-S_{\text {eff }} & =\ln \int\left[d U_{i}\right] \exp \left[\frac{\beta}{2 N} \sum_{p}\left(\operatorname{tr} U_{p}+\operatorname{tr} U_{p}^{\dagger}\right)\right] \equiv \lambda_{1} S_{1}+\lambda_{2} S_{2}+\ldots
\end{aligned}
$$

We expand around $\beta=0$ and arrange the effective couplings $\lambda_{n}=\lambda_{n}\left(\beta, N_{\tau}\right)$ in increasing order in $\beta$ of their leading terms. Thus, the $\lambda_{n}$ become less important the higher $n$. As we shall see, the interaction terms $S_{n}$ depend only on Polyakov loops

$$
L_{j} \equiv \operatorname{tr} W_{j} \equiv \operatorname{tr} \prod_{\tau=1}^{N_{\tau}} U_{0}\left(\vec{x}_{j}, \tau\right)
$$

With sufficiently accurate knowledge of the relations $\lambda_{n}\left(\beta, N_{\tau}\right)$, we are able to convert the couplings of the three-dimensional theory to those of the full theory. Determining the critical parameters $\lambda_{n, c}$ of the effective theory then gives a whole array of critical $\beta_{c}\left(N_{\tau}\right)$ for - in principle - all $N_{\tau}$. In the following we calculate strong coupling, i.e. small $\beta$, expansions of the leading $\lambda_{n}$.

Since the calculations are quite similar for different numbers of colours, we now specialise our derivation to the simpler case of $S U(2)$ and later provide the necessary changes for $S U(3)$. For more details see [18]. Using the character expansion as described e.g. in [19, 20], the effective action according to Eq. (2.2) can be written as

$$
-S_{\mathrm{eff}}=\ln \int\left[d U_{i}\right] \prod_{p}\left[1+\sum_{r \neq 0} d_{r} a_{r}(\beta) \chi_{r}\left(U_{p}\right)\right],
$$

where the sum extends over all irreducible representations $r$ with dimension $d_{r}$ and character $\chi_{r}$. The expansion coefficients $a_{r}(\beta)$ are accurately known [19] and in the following we use $u \equiv a_{f}$ as expansion parameter instead of $\beta$ for its better apparent convergence. The logarithm in this definition allows us to use the method of moments and cumulants [21], and we get the following cluster expansion

$$
\begin{aligned}
-S_{\mathrm{eff}} & =\sum_{C=\left(X_{l}^{n_{l}}\right)} a(C) \prod_{l} \Phi\left(X_{l} ;\left\{W_{j}\right\}\right)^{n_{l}} ; \\
\Phi\left(X_{l} ;\left\{W_{j}\right\}\right) & =\int\left[d U_{i}\right] \prod_{p \in X_{l}} d_{r_{p}} a_{r_{p}} \chi_{r_{p}}\left(U_{p}\right),
\end{aligned}
$$

where the combinatorial factor $a(C)$ equals 1 for a single polymer $X_{i}$ and -1 for two non-identical connected polymers. For clusters consisting of more than two polymers, $a(C)$ depends on how these polymers are connected. Our task is then to group together all graphs yielding the same interaction terms up to some order in $\beta$, and this finally gives the strong coupling expansion of the corresponding effective coupling $\lambda_{n}$. 


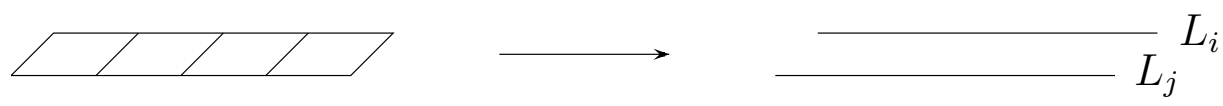

Figure 1: First graph with a nontrivial contribution after spatial integration for a lattice with temporal extent $N_{\tau}=4$. Four plaquettes in the fundamental representation lead to an interaction term involving two adjacent fundamental Polyakov loops $L_{i}$ and $L_{j}$.

\subsection{Leading order effective action}

The leading order result of the effective action has first been calculated in [10] and corresponds to a sequence of $N_{\tau}$ plaquettes that wind around the lattice in temporal direction, cf. Fig. 1. Its contribution is given by:

$$
\lambda_{1} S_{1}=u^{N_{\tau}} \sum_{<i j>} L_{i} L_{j}
$$

Hence, to leading order the first coupling of the effective theory is $\lambda_{1}\left(u, N_{\tau}\right)=u^{N_{\tau}}$.

For additional terms of the series for $\lambda_{1}$, we can use most of the graphs that also appear in the strong coupling expansion of the Polyakov loop susceptibility [23]. These corrections involve additional plaquettes, are hence of higher order in $u$ and we call these attached plaquettes decorations. Carrying out the calculations, we get the following results through order $u^{10}$ in the corrections relative to the leading order graph:

$$
\begin{aligned}
\lambda_{1}(u, 2) & =u^{2} \exp \left[2\left(4 u^{4}-8 u^{6}+\frac{134}{3} u^{8}-\frac{49044}{405} u^{10}\right)\right], \\
\lambda_{1}(u, 3) & =u^{3} \exp \left[3\left(4 u^{4}-4 u^{6}+\frac{128}{3} u^{8}-\frac{36044}{405} u^{10}\right)\right], \\
\lambda_{1}(u, 4) & =u^{4} \exp \left[4\left(4 u^{4}-4 u^{6}+\frac{140}{3} u^{8}-\frac{37664}{405} u^{10}\right)\right], \\
\lambda_{1}\left(u, N_{\tau} \geq 5\right) & =u^{N_{\tau}} \exp \left[N_{\tau}\left(4 u^{4}-4 u^{6}+\frac{140}{3} u^{8}-\frac{36044}{405} u^{10}\right)\right] .
\end{aligned}
$$

For smaller $N_{\tau}$ some graphs do not contribute since the temporal extent of their decoration is $\geq N_{\tau}$ so that they do not fit into the lattice.

\subsection{Higher order terms}

There occur several types of higher order graphs: larger numbers of loops involved, Polyakov loops at distances larger than one and Polyakov loops in higher dimensional representations. We begin by considering powers of the leading order term. Inspection of higher order terms shows that one can arrange a subclass of these terms in the following manner

$$
\sum_{<i j>}\left(\lambda_{1} L_{i} L_{j}-\frac{\lambda_{1}^{2}}{2} L_{i}^{2} L_{j}^{2}+\frac{\lambda_{1}^{3}}{3} L_{i}^{3} L_{j}^{3}-\ldots\right)=\sum_{<i j>} \ln \left(1+\lambda_{1} L_{i} L_{j}\right) .
$$


To see this, one calculates the corresponding graphs with $L_{i}^{2} L_{j}^{2}$ or $L_{i}^{3} L_{j}^{3}$, and the combinatorial factor $a(C)$ of Eq. (2.6) gives the correct prefactors for the series to represent a logarithm.

Next, let us consider couplings pertaining to next-to-nearest neighbour interactions. These appear once additional plaquettes are taken into account. Naively, the leading contribution should correspond to a planar graph with Polyakov loops at distance two. However, this graph is precisely cancelled by the contribution of the nearest-neighbour graph squared and its associated combinatorial factor -1 . The leading non-zero contribution therefore comes from L-shaped graphs and is given by

$$
\lambda_{2}\left(u, N_{\tau}\right) S_{2}=N_{\tau}\left(N_{\tau}-1\right) u^{2 N_{\tau}+2} \sum_{[k l]} L_{k} L_{l}
$$

where we have two additional spatial plaquettes and we sum over all pairs of loops with a diagonal distance of $\sqrt{2} a$, abbreviated by $[k l]$. With the same steps leading to Eq. (2.8), we finally arrive at the $S U(2)$ partition function

$$
Z=\int[d W] \prod_{<i j>}\left[1+\lambda_{1} L_{i} L_{j}\right] \prod_{[k l]}\left[1+\lambda_{2} L_{k} L_{l}\right]
$$

Finally, we include some remarks about the Polyakov loops in higher dimensional representations. Consider, e.g., the adjoint Polyakov loop: the leading order term emerging from a strong coupling expansion is

$$
\lambda_{a} S_{a}=v^{N_{\tau}} \sum_{<i j>} \chi_{a}\left(W_{i}\right) \chi_{a}\left(W_{j}\right), \quad v=\frac{2}{3} u^{2}+\frac{2}{9} u^{4}+\frac{16}{135} u^{6}+\ldots
$$

and hence $\lambda_{a} \sim u^{2 N_{\tau}}$, which is formally of lower order than the coupling $\lambda_{2}$. To next-to-leading order (valid for all $N_{\tau} \geq 2$ ) we have

$$
\lambda_{a}=v^{N_{\tau}}\left(1+N_{\tau} \frac{8}{3} \frac{u^{6}}{v}+\ldots\right)
$$

Effects of higher representations have also been investigated in the literature [14, 15, 25].

\subsection{The effective action for $S U(3)$}

In the case of $S U(3)$ the same steps as for $S U(2)$ apply. The only difference we have to keep in mind is that $S U(3)$ also has an anti-fundamental representation and consequently there is also a complex conjugate Polyakov loop variable $L_{i}^{*}$. Thus we get the one-coupling and two-coupling partition functions

$$
\begin{aligned}
& Z_{1}=\int[d W] \prod_{<i j>}\left[1+\lambda_{1}\left(L_{i} L_{j}^{*}+L_{i}^{*} L_{j}\right)\right], \\
& Z_{2}=\int[d W] \prod_{<i j>}\left[1+\lambda_{1}\left(L_{i} L_{j}^{*}+L_{i}^{*} L_{j}\right)\right] \prod_{[k l]}\left[1+\lambda_{2}\left(L_{k} L_{l}^{*}+L_{k}^{*} L_{l}\right)\right] .
\end{aligned}
$$


The effective coupling $\lambda_{1}\left(u, N_{\tau}\right)$ is obtained as (for this gauge group we consider only even values of $N_{\tau}$ ):

$$
\begin{gathered}
\lambda_{1}(2, u)=u^{2} \exp \left[2 \left(4 u^{4}+12 u^{5}-18 u^{6}-36 u^{7}\right.\right. \\
\left.\left.+\frac{219}{2} u^{8}+\frac{1791}{10} u^{9}+\frac{830517}{5120} u^{10}\right)\right], \\
\lambda_{1}(4, u)=u^{4} \exp \left[4 \left(4 u^{4}+12 u^{5}-14 u^{6}-36 u^{7}\right.\right. \\
\left.\left.+\frac{295}{2} u^{8}+\frac{1851}{10} u^{9}+\frac{1035317}{5120} u^{10}\right)\right], \\
\lambda_{1}\left(N_{\tau} \geq 6, u\right)=u^{N_{\tau}} \exp \left[N _ { \tau } \left(4 u^{4}+12 u^{5}-14 u^{6}-36 u^{7}\right.\right. \\
\left.\left.+\frac{295}{2} u^{8}+\frac{1851}{10} u^{9}+\frac{1055797}{5120} u^{10}\right)\right] .
\end{gathered}
$$

For the first terms of the next-to-nearest neighbour coupling $\lambda_{2}\left(N_{\tau}, u\right)$ we find

$$
\begin{aligned}
\lambda_{2}(2, u) & =u^{4}\left[2 u^{2}+6 u^{4}+31 u^{6}\right], \\
\lambda_{2}(4, u) & =u^{8}\left[12 u^{2}+26 u^{4}+364 u^{6}\right], \\
\lambda_{2}(6, u) & =u^{12}\left[30 u^{2}+66 u^{4}\right], \\
\lambda_{2}\left(N_{\tau} \geq 8, u\right) & =u^{2 N_{\tau}}\left[N_{\tau}\left(N_{\tau}-1\right) u^{2}\right],
\end{aligned}
$$

while the leading coupling of adjoint loops is (valid for $N_{\tau} \geq 2$ )

$$
\lambda_{a}=v^{N_{\tau}}\left(1+N_{\tau} \frac{3}{2} \frac{u^{6}}{v}+\ldots\right), \quad v=\frac{9}{8} u^{2}-\frac{9}{8} u^{3}+\frac{81}{32} u^{4}+\ldots
$$

\section{Numerical simulation of the effective theories}

\subsection{The one coupling model}

For the purpose of numerical simulations, a further simplification is achieved by using the trace of the Polyakov loops for the path integral measure as degrees of freedom (complex numbers, $\left|L_{x}\right| \leq 3$, instead of matrices), and rewrite the one-coupling partition function for $S U(3)$, Eq. (2.12),

$$
Z=\left(\prod_{x} \int \mathrm{d} L_{x}\right) e^{-S_{\text {eff }}} ; S_{\text {eff }}=-\sum_{<i j>} \log \left(1+2 \lambda_{1} \operatorname{Re} L_{i} L_{j}^{*}\right)-\sum_{x} V_{x}
$$

The potential term $V_{x}$ is the Jacobian induced by the Haar measure of the original group integration; rotating the matrices to the diagonal form $\operatorname{diag}\left(e^{i \theta}, e^{i \phi}, e^{-i(\theta+\phi)}\right)$, with $|\theta|,|\phi| \leq \pi$, we have [26]:

$$
V_{x}=\frac{1}{2} \log \left(27-18\left|L_{x}\right|^{2}+8 \operatorname{Re} L_{x}^{3}-\left|L_{x}\right|^{4}\right) .
$$

The integration measure actually used in our simulation then takes the form

$$
\int \mathrm{d} L_{x} e^{V_{x}}=\int_{-\pi}^{+\pi} \mathrm{d} \phi_{x} \int_{-\pi}^{+\pi} \mathrm{d} \theta_{x} e^{V_{x}}
$$

When working on the $S U(2)$ theory, $-2 \leq L_{x} \leq+2$ is a real number and we simply have

$$
\int_{-2}^{+2} \mathrm{~d} L_{x} e^{V_{x}}, V_{x}=\frac{1}{2} \log \left(4-L_{x}^{2}\right) \text {. }
$$



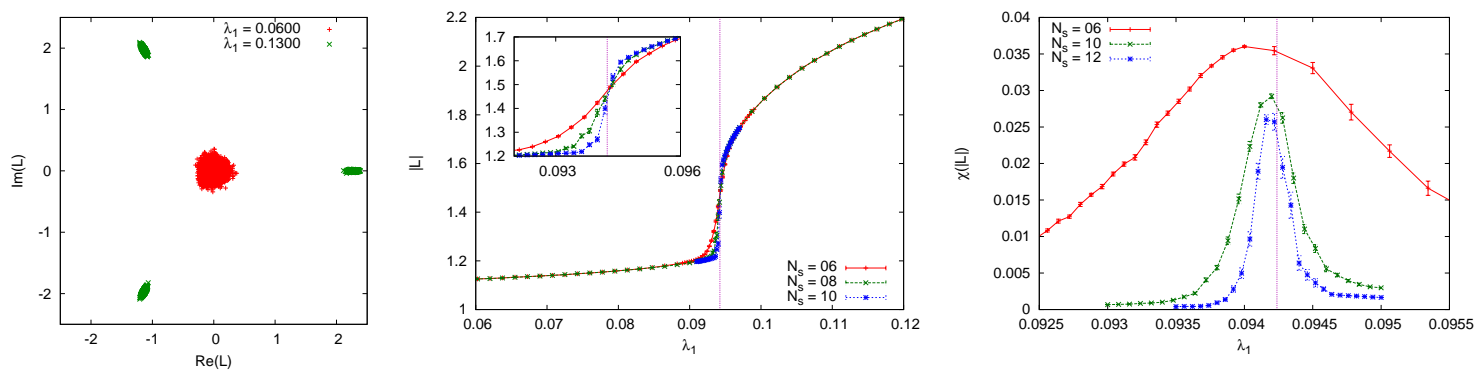

Figure 2: Left: Distribution of $L$ for small and large $\lambda_{1}$ on a lattice with $N_{s}=6$ and $M=1$. Middle, Right: Expectation value of $|L|$ and its susceptibility. The vertical line marks the infinite-volume transition.

\subsection{A "sign problem" and its solution}

Our numerical approach will be a straightforward Metropolis local update algorithm; however, the Boltzmann weights to consider are in the form $\exp \left(\log \left(1+2 \lambda_{1} \operatorname{Re}\left(L_{i} L_{j}^{*}\right)\right)\right)$ : for high enough couplings, they can be also negative, thus spoiling the update technique (the partition function being, overall, still positive). In the $S U$ (2) case, the threshold coupling $\lambda^{T}=1 / 4$ is well beyond the phase transition, so that in practice there is no problem around criticality, but the $S U(3)$ threshold of $1 / 9$ is very close to the transition and a direct numerical investigation of the model as in Eq. (3.1) is impossible.

Our approach to overcome this problem is the following: we Taylor-expand the logarithm in the effective action to some order $M$ in powers of $q \equiv \lambda_{1} \operatorname{Re} L_{i} L_{j}^{*}$ (undoing the resummation as in Eq. (2.8)), obtaining models free of the problem:

$$
S_{\mathrm{eff}}^{(M)}=-\sum_{x} V_{x}-\sum_{<i j>}\left(2 q-2 q^{2}+\frac{8}{3} q^{3}-4 q^{4}+\frac{32}{5} q^{5}-\ldots-(-1)^{M} \frac{2^{M}}{M} q^{M}\right) .
$$

In this way we can identify a critical point for each $M$ and look for their convergence as $M \rightarrow \infty$. Also, we can compare to the $S U(2)$ case where the $M=\infty$ value is directly calculable.

\subsection{Phase structure, critical coupling and finite size analysis}

Our first task is to establish the phase structure of the effective theory, where we focus on the physically interesting case of $S U(3)$. Based on the global $Z(3)$ symmetry of the model, one expects spontaneous breaking of that symmetry for some critical value of the coupling $\lambda_{1, c}$. Fig. 2 shows the behaviour of the field variable $L$ as a function of $\lambda_{1}$. As expected from the $4 \mathrm{~d}$ parent theory, there is indeed a transition from a disordered or mixed phase, with values of $L$ scattering about zero, to an ordered phase at large coupling where the three $Z(3)$-phases are populated separately. In the thermodynamic limit, one of these vacua will be chosen and the symmetry is broken spontaneously, $\langle L\rangle=0$ for $\lambda_{1}<\lambda_{1, c}$ and $\langle L\rangle \neq 0$ for $\lambda_{1}>\lambda_{1, c}$. Correspondingly, the expectation value of $|L|$ rises abruptly at some critical coupling $\lambda_{1, c}$, as shown in Fig. 目 (middle). On a finite size lattice, the phase transition is smoothed out, non-analyticities are approached gradually with growing volume, as the figure illustrates.

The critical coupling, $\lambda_{1, c}$, is located via finite-size scaling. After identifying a pseudo-critical $\lambda_{1, c}\left(N_{s}\right)$ for a number of finite systems, the relation

$$
\lambda_{1, c}\left(N_{s}\right)=\lambda_{1, c}+b N_{s}^{-1 / v}
$$



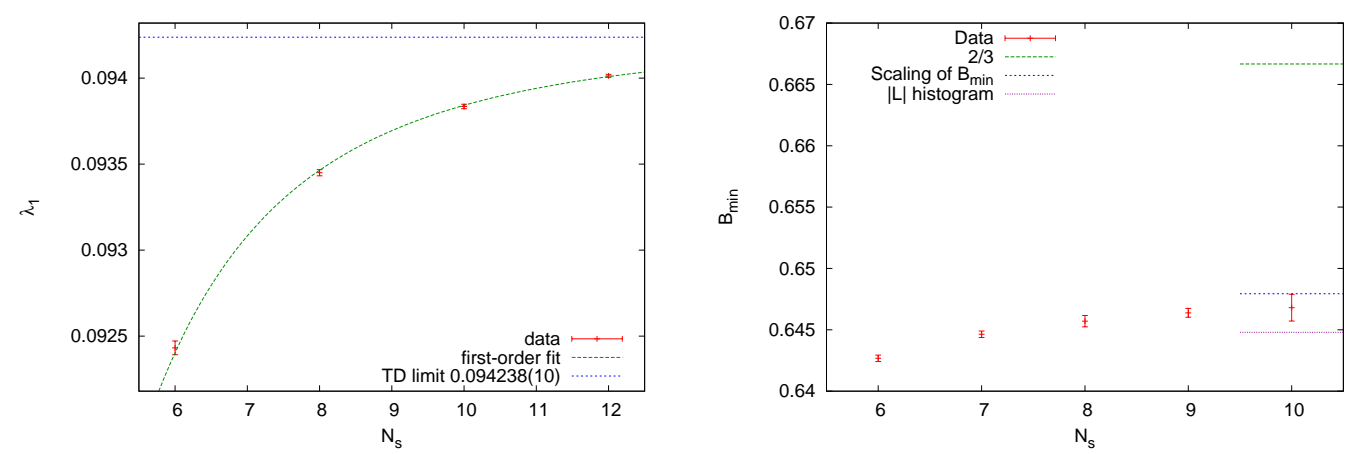

Figure 3: Left: Position of the minimum of the Binder cumulant $B(E)$ for $S U(3), M=1$, for different lattice sizes. The horizontal line is the thermodynamic limit resulting from the fit to Eq. (3.6). Right: Behaviour of $B_{\min }\left(N_{s}\right)$, along with its thermodynamic limit obtained with the $\mathscr{O}\left(N_{s}^{-3}\right)$ scaling law and the independent estimate $B_{\infty}$ from the $|L|$ histogram. Also the second-order limit value $2 / 3$ is shown.

is used, with $v=1 / 3$ for the $S U$ (3) first-order transitions and, in the $S U(2)$ case, the $3 \mathrm{~d}$ Ising value $v=0.63002$ [27]. Numerically, we found satisfactory results with data produced in just a few days on a desktop PC.

For the definition of the pseudo-critical coupling, one can look at the energy $E=-S_{\text {eff }} / \lambda_{1}$ (neglecting the potential term) or derived quantities, but in general, due to the nonlinearity of $S_{\text {eff }}$ in the coupling, we preferred to look at the average modulus $|L|$; one can then define $\lambda_{1, c}$ as the minimum/maximum of the associated Binder cumulant/susceptibility, which indeed featured a more robust scaling:

$$
B(|L|)=1-\frac{\left\langle|L|^{4}\right\rangle}{3\left\langle|L|^{2}\right\rangle^{2}} \quad ; \quad \chi(|L|)=\left\langle(|L|-\langle|L|\rangle)^{2}\right\rangle .
$$

\subsection{Critical coupling and order of the transition for $S U(3)$}

The truncated theories with $M=1,3,5$ were simulated on lattices with spatial sizes $N_{s}=$ $6,8,10$ (plus $N_{s}=12$ for the $M=1$ theory). For each volume, $\sim 30$ values of the couplings are sampled by $\sim 10^{6}$ update sweeps each. Measurements were taken every $\sim 30$ updates.

Regardless of the truncation order $M$, the $S U(3)$ theories display a first-order transition; among the associated features, we found very long thermalisation times $\propto \exp \left(c N_{s}^{3}\right)$ as is expected for tunnelling phenomena (Fig. Đ): for instance, a system with size $N_{s}=16$ would require, around criticality, $\sim 10^{6}$ update sweeps to thermalise.

First we consider the model with $M=1$. The first-order nature of the transition is established by fitting the pseudo-critical couplings to the scaling law, Eq. (3.6), with $v=1 / 3$, see Fig. 3 (left). The behaviour of the minimum $B_{\min }$ of $B(|L|)$ is a further confirmation; this quantity, as demonstrated in [28, 29], scales as $B_{\min }\left(N_{s}\right)=B_{\infty}+B^{(2)} N_{s}^{-3}+\mathscr{O}\left(N_{s}^{-6}\right)$, with a thermodynamic limit which is smaller than the second-order value $2 / 3$,

$$
B_{\infty}=\frac{2}{3}-\frac{1}{12}\left(\frac{|L|_{1}}{|L|_{2}}-\frac{|L|_{2}}{|L|_{1}}\right)^{2}
$$



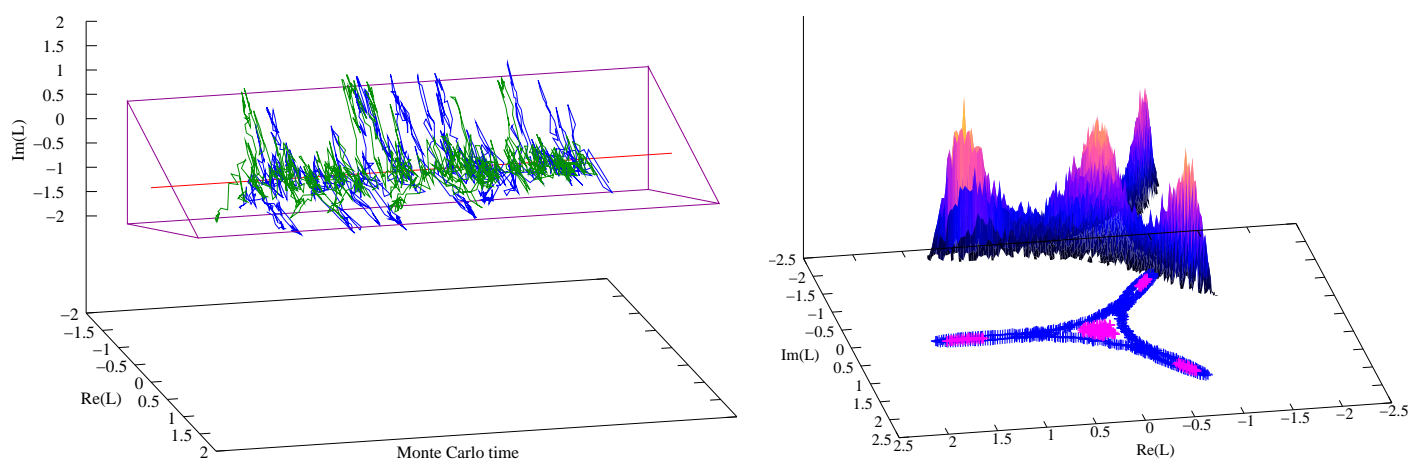

Figure 4: Left: Behaviour of $L$ with Monte Carlo time for two $N_{s}=6$ trajectories in the $S U(3) M=1$ theory with $\lambda_{1}=0.0935$. Right: Histogram for $L$, obtained from 60 such trajectories. The tunnelling between the central and the three broken-symmetry vacua is apparent.
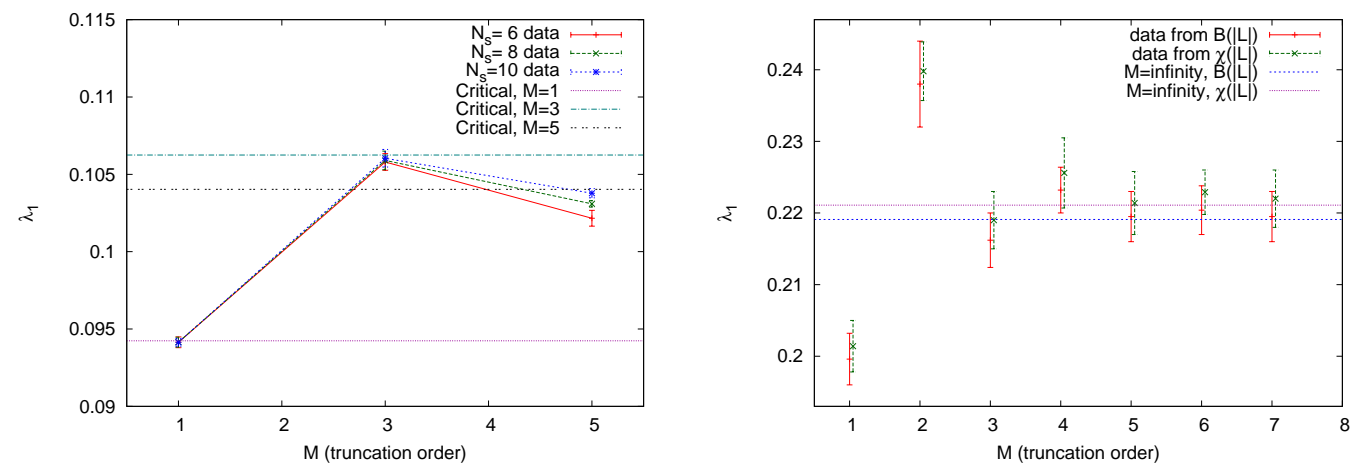

Figure 5: Truncation-dependence of the critical points. Left: in the $S U(3)$ case, the data points refer to three system sizes, while the lines mark the extrapolated critical points; the latter are found at $\lambda_{1, c}=0.094238(10), 0.10635(11), 0.10403(28)$ for $M=1,3,5$ respectively. Right: for $S U(2)$, pseudocritical points for $N_{s}=8$ obtained from $B(|L|)$ and $\chi(|L|)$ for a variety of truncations (data points) and compared with the untruncated $M=\infty$ values (lines).

with $|L|_{1}$ and $|L|_{2}$ the two local maxima of the $|L|$ double-peaked histogram. A direct comparison between the results for $B_{\infty}$ from scaling analysis and from the location of $|L|_{i}$ shows an agreement within two standard deviations, the residual discrepancy being probably due to neglecting higherorder $N_{s}^{-6}$ corrections.

In the next step we need to investigate the behaviour of the models with higher $M$. Again we observe first order transitions, which become sharper with increasing $M$. Moreover, finitesize effects are stronger for higher $M$, Fig. 5 (left). The critical couplings identified for the $M=$ $1,3,5$ effective theories in the thermodynamic limit are also quoted there. Judging from these three values, the series seems to be rapidly converging, with only $\sim 3 \%$ difference between $M=$ 3,5. The residual difference between this estimate and the $M=\infty$ critical coupling is completely subdominant compared to the other systematic errors contributing to the final results. Also, the direct comparison with the $S U(2)$ case below, where the $M=\infty$ data are directly available, supports a rapid convergence, Fig. 5 (right). 


\subsection{Critical coupling and order of the transition for $S U(2)$}

In this family of theories the transition is second-order; with much less relaxation problems (e.g. 4000 steps for $N_{s}=16$ ), larger lattices (up to $N_{s}=28$ ) were available. With the same approach as for $S U(3)$, the nature of the transition was confirmed by: (a) $\lambda_{1, c}\left(N_{s}\right)$ scaling with the $3 \mathrm{~d}$ Ising critical index, (b) Binder cumulant analysis approaching 2/3 for large systems, and (c) $|L|$ histogram inspection, where a single peak continuously moves to the right as the coupling is increased. All inspected values of $M$ yielded the same features. Moreover, here a direct comparison with the $M=\infty$ untruncated model is possible, and shows that a rapid convergence is indeed realised (Fig. 5, right); in particular, we found

$$
\lambda_{1, c}(M=1)=0.195374(42) ; \lambda_{1, c}(M=\infty)=0.21423(70),
$$

which indicates quite small systematic deviations due to choosing one particular truncation.

\subsection{Two-coupling models for $S U(3)$}

In this section we study the influence of including a second coupling. We consider two possibilities: the first one is switching on the interaction between next-to-nearest neighbours. The $S U(3)$ version of Eq. (2.10) reads:

$$
Z=\left(\prod_{x} \int \mathrm{d} L_{x}\right) \prod_{<i j>}\left(1+2 \lambda_{1} \operatorname{Re} L_{i} L_{j}^{*}\right) \prod_{[k l]}\left(1+2 \lambda_{2} \operatorname{Re} L_{k} L_{l}^{*}\right) e^{\sum_{x} V_{x}} .
$$

We remark that now there are two terms suffering from the above-mentioned sign problem: a truncated expansion is then needed in both, and the two truncation parameters $\left(M_{1}, M_{2}\right)$ should be chosen in a consistent way, for all $N_{\tau}$, with respect to the power in $u$ we want to keep. We adopted the choice $(3,1)$ after checking numerically that higher values of $M_{2}$ give negligible differences in the results.

In the other model, we allow the nearest neighbours to interact also in the adjoint representation as described before. The partition function in this case (with the adjoint part already truncated at $\left.M_{2}=1\right)$ is given by

$$
Z=\left(\prod_{x} \int \mathrm{d} L_{x}\right) \prod_{<i j>}\left(1+2 \lambda_{1} \operatorname{Re} L_{i} L_{j}^{*}\right) \prod_{<i j>} e^{\lambda_{a}\left(\operatorname{Tr}^{(a)} W_{i}\right)\left(\operatorname{Tr}^{(a)} W_{j}\right)} e^{\Sigma_{x} V_{x}},
$$

with the adjoint trace $\operatorname{Tr}^{(a)} W=|\operatorname{Tr} W|^{2}-1$. Also in this case, the truncation $(3,1)$ was employed.

In these two-dimensional parameter spaces, there is a critical line separating the symmetric and the broken phases. However, for a given $N_{\tau}$, only a one-dimensional manifold in this space represents the image of the original gauge theory, since both couplings are functions of the sole $u$. The strategy was then to identify the shape of the critical line and find, for each temporal lattice extent, the intersection with the curve enforcing that particular value of $N_{\tau}$.

In both models, the critical lines were found by interpolation after locating 11 critical points at as many fixed values of the second coupling; it turned out that a linear parametrisation was good enough in describing them (within our precision, finite-size effects were practically invisible):

$$
\begin{aligned}
& \lambda_{1, c}=a+b \lambda_{2} \quad \text { with } \quad a=0.10628(8), b=-1.891(4) . \\
& \lambda_{1, c}=a+b \lambda_{a} \quad \text { with } \quad a=0.10637(15), b=-1.422(22) .
\end{aligned}
$$



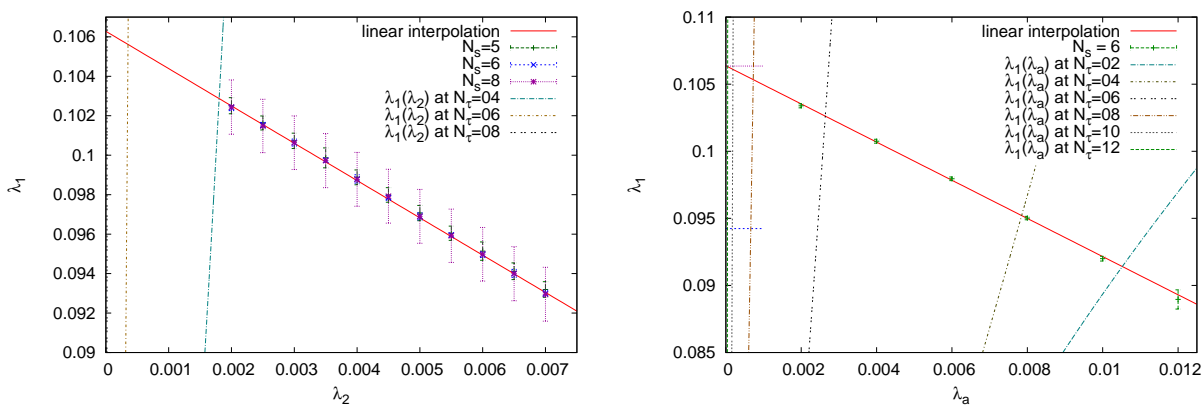

Figure 6: Critical line in the two-coupling space, determined from $\chi(|L|)$. Dashed lines give the parameter space representing a $4 \mathrm{~d}$ theory with fixed $N_{\tau}$. Left: $\left(\lambda_{1}, \lambda_{2}\right)$. Right: $\left(\lambda_{1}, \lambda_{a}\right)$.

\begin{tabular}{|c||c|c||c|}
\hline$N_{\tau}$ & $M=1$ & $M=\infty$ & 4d YM \\
\hline 3 & $2.15537(89)$ & $2.1929(13)$ & $2.1768(30)$ \\
4 & $2.28700(55)$ & $2.3102(08)$ & $2.2991(02)$ \\
5 & $2.36758(40)$ & $2.3847(06)$ & $2.3726(45)$ \\
6 & $2.41629(32)$ & $2.4297(05)$ & $2.4265(30)$ \\
8 & $2.47419(22)$ & $2.4836(03)$ & $2.5104(02)$ \\
12 & $2.52821(14)$ & $2.5341(02)$ & $2.6355(10)$ \\
16 & $2.55390(10)$ & $2.5582(02)$ & $2.7310(20)$ \\
\hline
\end{tabular}

Table 1: Critical couplings $\beta_{c}$ for $S U(2)$ from two effective theories compared to simulations of the $4 \mathrm{~d}$ theory [31, 30, 32]).

The value of $a$ was always, as expected, compatible with the estimate for the critical point of the $M=3$ one-coupling theory.

By plotting these critical lines and the family of curves coming from requiring a given $N_{\tau}$, one sees that the latter accumulate towards vanishing second-coupling as $N_{\tau}$ increases (Fig. 6): this implies that the effect of including those interactions is less and less important at finer lattice spacings: only at very low values of temporal extent does the inclusion of a second coupling make any visible difference.

\section{Mapping back to 4d Yang-Mills}

Having established the critical couplings for our effective theories and tested their reliability, we are now ready to map them back to the original thermal Yang-Mills theories by using Eqs. (2.7, 2.14). In Tables 1, 2 2 we collect the values for the critical gauge couplings, $\beta_{c}$, obtained in this way from the effective theories and compare them to the values obtained from simulations of the full $4 \mathrm{~d}$ theories for $S U(2), S U(3)$, respectively.

The agreement is remarkable in all cases, with the relative error of the effective theory results compared to the full ones shown in Fig. ㄱ. The comparison of alternative truncations of the logarithm shows once more that it has almost no influence on the accuracy of the final result, as described earlier. Interestingly, there appears to be a 'region of best agreement', with the deviation 


\begin{tabular}{|c||c|c|c|c||c|}
\hline$N_{\tau}$ & $M=1$ & $M=3$ & $M_{1}, M_{2}\left(\lambda_{2}\right)=3,1$ & $M_{1}, M_{2}\left(\lambda_{a}\right)=3,1$ & $4 \mathrm{~d} \mathrm{YM}$ \\
\hline 4 & 5.768 & 5.830 & 5.813 & 5.773 & $5.6925(002)$ \\
6 & 6.139 & 6.173 & 6.172 & 6.164 & $5.8941(005)$ \\
8 & 6.300 & 6.324 & 6.324 & 6.322 & $6.0010(250)$ \\
10 & 6.390 & 6.408 & 6.408 & 6.408 & $6.1600(070)$ \\
12 & 6.448 & 6.462 & 6.462 & 6.462 & $6.2680(120)$ \\
14 & 6.488 & 6.500 & 6.500 & 6.500 & $6.3830(100)$ \\
16 & 6.517 & 6.528 & 6.528 & 6.528 & $6.4500(500)$ \\
\hline
\end{tabular}

Table 2: Critical couplings $\beta_{c}$ for $S U$ (3) from different effective theories compared to simulations of the $4 \mathrm{~d}$ theory [3], 33]).
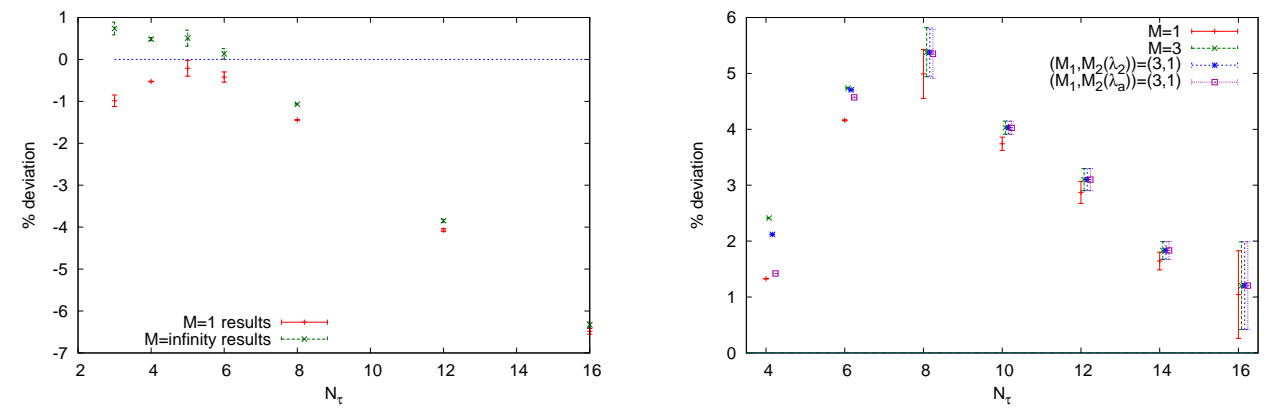

Figure 7: Relative error of $\beta_{c}$ predicted by the effective theories when compared to simulations of the $4 \mathrm{~d}$ theories, for $S U(2)$ (left) and $S U(3)$ (right).

growing both for small and large $N_{\tau}$. We ascribe this to the fact that there are two competing systematic errors, as discussed earlier: the validity of the strong coupling series for a given coupling $\lambda_{i}$ is better the smaller $\beta$ and hence $N_{\tau}$, whereas the truncation of the next-to-nearest neighbour interactions gains validity with growing $N_{\tau}$. In particular in the case of $S U(3)$, there appears to be a cancellation of the two kinds of systematics, rendering the effective description better for the original theory on finer lattices.

The strong-coupling series was inspected both by comparing the resulting $\beta_{c}$ from series of different depth and by Padé analysis, and we observe a satisfactory convergence. It was also found that the error due to the truncation of the strong-coupling series is much larger than that from neglecting higher couplings.

One can also compare the results presented here with those from the inverse Monte Carlo approach, where the effective theory is found in a completely non-perturbative way; inspection of the $S U$ (2) case [14], in particular, shows that the abrupt change of curvature in the inverse Monte Carlo function $\lambda_{1}(\beta)$ at the critical point is not captured by our strong-coupling approach, which is consistent with $\beta_{c}$ marking the radius of convergence also for the series expansion of the effective coupling $\lambda_{1}$. Thus, the inverse Monte Carlo approach has a wider range of validity whereas the series approach furnishes analytically known mappings between the full and effective theories. 


\section{Conclusions}

We have derived, by means of strong coupling expansions, an effective description for lattice pure gauge theories at finite temperature which respects explicitly the requirement of centre symmetry and has only scalar Polyakov loop variables as degrees of freedom. Moreover, due to the dimensional reduction involved, the $N_{\tau}$-dependence is encoded completely in the maps from the effective to the original coupling $\beta$, whose expansion can be extended, in principle, to higher orders. We have also considered interaction terms other than the leading one, namely a next-to-nearest neighbour interaction and an adjoint-representation coupling term.

Our Monte Carlo approach to the models, while requiring modest computational resources, confirms the expected nature of the symmetry-breaking transition for both $S U(2)$ - and $S U(3)$-based effective formulations, and allowed us to predict the critical point $\beta_{c}$ of the original $4 \mathrm{~d}$ thermal gauge theories with an accuracy within a few percent for a variety of values of $N_{\tau}$. Particular attention was devoted to estimating the effect of employing different approximations, with quite stable answers in support of the good convergence of the series, and of neglecting higher-order interaction terms, which again does not have a strong effect on the final answers especially at finer lattices.

An extension of the present work could be the study of $S U(N)$ gauge theories with $N>3$ (cf. [34] and references therein), which can be performed much in the same way as the cases examined here; even more intriguing is the possibility to keep the theory simple while getting a step closer to physical QCD, i. e. by introducing fermions and finite baryon density, for instance by employing a hopping parameter expansion [23, 35].

\section{Acknowledgements}

S. L. and O. P. are partially supported by the German BMBF grant FAIR theory: the QCD phase diagram at vanishing and finite baryon density, 06MS9150, and by the Helmholtz International Center for FAIR within the LOEWE program of the State of Hesse. J. L. acknowledges financial support by the EU project Study of Strongly interacting Matter, No. 227431, and by the BMBF under the project Heavy Quarks as a Bridge between Heavy Ion Collisions and QCD, 06BI9002.

\section{References}

[1] A. D. Linde, Phys. Lett. B 96 (1980) 289.

[2] P. H. Ginsparg, Nucl. Phys. B 170 (1980) 388;

[3] T. Appelquist and R. D. Pisarski, Phys. Rev. D 23 (1981) 2305.

[4] K. Kajantie, M. Laine, K. Rummukainen and M. E. Shaposhnikov, Nucl. Phys. B 503 (1997) 357 [arXiv:hep-ph/9704416].

[5] A. Vuorinen and L. G. Yaffe, Phys. Rev. D 74 (2006) 025011 [arXiv:hep-ph/0604100].

[6] R. D. Pisarski, Phys. Rev. D 74 (2006) 121703 [arXiv:hep-ph/0608242]. 
[7] Ph. de Forcrand, A. Kurkela and A. Vuorinen, Phys. Rev. D 77 (2008) 125014 [arXiv:0801.1566 [hep-ph]].

[8] A. Dumitru, D. Smith Phys. Rev. D 77 (2008) 094022 [arXiv:0711.0868v1 [hep-lat]].

[9] B. Svetitsky and L. G. Yaffe, Nucl. Phys. B 210 (1982) 423.

[10] J. Polonyi and K. Szlachanyi, Phys. Lett. B 110 (1982) 395.

[11] F. Green and F. Karsch, Nucl. Phys. B 238 (1984) 297.

[12] A. Gocksch and M. Ogilvie, Phys. Rev. D 31 (1985) 877.

[13] M. Gross and J. F. Wheater, Nucl. Phys. B 240 (1984) 253.

[14] T. Heinzl, T. Kaestner and A. Wipf, Phys. Rev. D 72 (2005) 065005 [arXiv:hep-lat/0502013].

[15] C. Wozar, T. Kaestner, A. Wipf and T. Heinzl, Phys. Rev. D 76 (2007) 085004 [arXiv:0704.2570 [hep-lat]].

[16] M. Billò, M. Caselle, A. D’Adda and S. Panzeri, Nucl. Phys. B 472 (1996) 163 [arXiv:hep-lat/9601020].

[17] T. Z. Nakano, K. Miura and A. Ohnishi, arXiv:1009.1518 [hep-lat].

[18] J. Langelage, S. Lottini and O. Philipsen, arXiv:1010.0951 [hep-lat].

[19] I. Montvay and G. Münster, Cambridge, UK: Univ. Pr. (1994) 491 p. (Cambridge monographs on mathematical physics).

[20] J. M. Drouffe and J. B. Zuber, Phys. Rept. 102, 1 (1983).

[21] G. Münster, Phys. Lett. B 95 (1980) 59.

[22] J. Langelage, G. Münster and O. Philipsen, JHEP 0807 (2008) 036 [arXiv:0805.1163 [hep-lat]].

[23] J. Langelage and O. Philipsen, JHEP 1001 (2010) 089 [arXiv:0911.2577 [hep-lat]].

[24] M. Mathur, arXiv:hep-lat/9501036.

[25] A. Dumitru, Y. Hatta, J. Lenaghan, K. Orginos and R. D. Pisarski, Phys. Rev. D 70 (2004) 034511 arXiv:hep-th/0311223.

[26] M. Gross, J. Bartholomew and D. Hochberg, Report No. EFI-83-35-CHICAGO, 1983.

[27] M. Hasenbusch, arXiv:1004.4486.

[28] J. Lee and J. M. Kosterlitz, Phys. Rev. B43 (1991) 3265.

[29] A. Billoire, T. Neuhaus and B. Berg, Nucl. Phys. B396 (1993) 779.

[30] I. L. Bogolubsky, V. K. Mitrjushkin, A. V. Sergeev, M. Müller-Preussker and H. Stüben, Nucl. Phys. Proc. Suppl. 129 (2004) 611.

[31] J. Fingberg, U. M. Heller and F. Karsch, Nucl. Phys. B 392 (1993) 493.

[32] A. Velytsky, Int. J. Mod. Phys. C 19, 1079 (2008) [arXiv:0711.0748 [hep-lat]].

[33] J. Kogut, M. Stone and H. W. Wyld, Phys. Rev. Lett. 50 (1983) 393.

[34] M. Panero, Phys. Rev. Lett. 103 (2009) 232001 [arXiv:0907.3719 [hep-lat]].

[35] J. Langelage and O. Philipsen, JHEP 1004 (2010) 055 [arXiv:1002.1507 [hep-lat]]. 